\title{
Extractivos del duramen de especies latífoliadas como preservantes en la madera de Pinus caribaea Morelet
}

\author{
Heartwood extractives of hardwood species as preservatives \\ in Pinus caribaea Morelet wood
}

\author{
Jesús Velásquez ${ }^{\circledR}$, Luis Rojas $^{\left({ }^{(0}\right.}$ y Osvaldo Encinas ${ }^{3^{(}}$
}

Velásquez, J., Rojas, L. y Encinas, O. (2019). Extractivos del duramen de especies latífoliadas como preservantes en la madera de Pinus caribaea Morelet. Colombia Forestal, 22(2), 44-54.

Recepción: 20 de noviembre de 2018

\section{Resumen}

Se evaluó el comportamiento de los extractos del duramen de tres especies latífoliadas: Handroanthus serratifolius, Centrolobium paraense y Tectona grandis frente a hongos de pudrición marrón y blanca. Se impregnaron bloques de albura de Pinus caribaea con extractos a tres niveles de concentración $0.2 \% 1 \%$ y $2 \%(\mathrm{p} / \mathrm{v})$. Los bloques tratados se expusieron la acción de Gloeophyllum trabeum y Trametes versicolor durante 16 semanas mediante el método de soil block, normas ASTM D 1413. Las soluciones al $1 \%$ y $2 \%$ fueron más eficientes en disminuir el ataque de los microorganismos de prueba. El extracto de H. serratifolius fue el más efectivo, seguido de C. paraense y con efectos más sutiles pero efectivos el extracto de $T$. grandis. Los resultados demostraron que los metabolitos secundarios del duramen son una fuente importante de moléculas bioactivas para el tratamiento de maderas menos resistentes como el $P$. caribaea.

Palabras clave: biopreservantes, degradación de madera, Gloeophyllum trabeum, Trametes versicolor
Aprobación: 4 de junio de 2019

\begin{abstract}
The performance of heartwood extracts of three durable hardwood species Handroanthus serratifolius Centrolobium paraense y Tectona grandis on brown and white rotter fungi were investigated. Extract dosages were $0.2 \% 1 \%$ y $2 \%(\mathrm{w} / \mathrm{v})$. Treated blocks were exposed to G. trabeum and T. versicolor attack for 16 weeks by the soil block method using the procedure set out in ASTM D 1413. Only extract solutions of $1 \%$ and $2 \%$ were found more efficient in suppressing fungal attack. The extract of $H$. serratifolius was more effective, followed by C. paraense and with more subtle but effective effects with $T$. grandis. The results showed that the secondary metabolites of the heartwood are an important source of bioactive molecules for the treatment of less resistant woods such as $P$. caribaea.
\end{abstract}

Key words: biopreservant, wood decay, GloeophyIlum trabeum, Trametes versicolor.

Centro Biotecnológico de Guayana, Laboratorio de Biotecnología de la Madera, Universidad Nacional Experimental de Guayana, Venezuela. jvelasquez@uneg.edu.ve.

Instituto de Investigaciones, Facultad de Farmacia y Bioanálisis, Universidad de Los Andes, Mérida, Venezuela. rojas|@ula.ve.

Laboratorio Nacional de Productos Forestales, Grupo en Conservación de Maderas GICOM, Facultad de Ciencias Forestales y Ambientales, Universidad de Los Andes, Mérida, Venezuela. osenbla@gmail.com. 


\section{INTRODUCCIÓN}

Para incrementar la vida en servicio, la manufactura, el transporte y el almacenamiento de la madera y de diversos productos maderables, estos deben ser tratados con productos químicos tóxicos a los microorganismos que causan su destrucción; estos productos son comúnmente conocidos como preservantes (Antwi y Damoah, 2010; González et al., 2015). Dentro de estos resaltan los fungicidas sintéticos como las sales a base de cobre-cromo-arsénico (CCA), cobre-cromo-boro (CCB) y cobre-amonio cuaternario (ACQ), entre otros. Estos, si bien garantizan una elevada protección de la madera, muestran el inconveniente de posible lixiviación, ocasionando la pérdida de algunos de sus componentes al medioambiente. Por otra parte, los problemas toxicológicos en la salud de muchas personas en contacto directo con ciertos productos químicos empleados en la industria de la preservación de la madera (pentaclorofenol, CCA, CCB, ACQ) e incluso con la madera ya tratada, hacen necesario el estudio y búsqueda de productos alternativos capaces de inhibir el desarrollo de los agentes causantes de la pudrición en la madera, pero que a su vez presenten una adecuada inocuidad ecológica y toxicológica (Peredo,1993; Schultz y Nicholas, 2000; 2002; Antwi y Damoah, 2010; Singh y Singh, 2012).

Muchas de las especies latífoliadas que presentan una transición abrupta albura-duramen poseen una particular bioresistencia al deterioro por acción de hongos, bacterias y al ataque de insectos. Esta resistencia se encuentra generalmente atribuida a la presencia, en las paredes celulares de las fibras, de sustancias químicas activas que desempeñan un papel importante en la durabilidad natural, ya que proporcionan propiedades fungicidas, fungistáticas, hidrofóbicas, quelantes y antioxidantes que eliminan o inhiben la actividad enzimática, el crecimiento y desarrollo de los agentes xilófagos (Hillis, 1987; Sjöström, 1981; Eaton y Hale, 1993; Suttie y Orsler, 1996; Schultz y Nicholas, 2000; 2002; Sen et al., 2009). Estos metabolitos secundarios o extractivos abarcan una serie de compuestos químicos como alcaloides, flavonoides, terpenos, terpenoides, estilbenos, lignanos, quinonas y otros compuestos fenólicos, los cuales, además de contribuir como mecanismo de defensa, son responsables de las propiedades organolépticas de la madera en cada especie (Sjöström, 1981; Fengel y Wegener, 1984; Hillis, 1987; Tsoumis, 1991; Tascioglu et al., 2013).

En la actualidad se ha intensificado la evaluación, extracción, elucidación y síntesis de compuestos extraídos del duramen de especies resistentes a hongos e insectos, los cuales podrían ser empleados como ingredientes activos en la formulación de eficientes y efectivos preservantes, para maderas de baja durabilidad natural, con un mínimo de efectos colaterales (Doppelreiter y Korioth, 1978; Dawson y Morrell, 1990; Goktas et al., 2007; Sá et al., 2009; Antwi y Damoah, 2010; González et al., 2015). La investigación en el área ha sido relativamente extensa, un resumen de las mismas puede consultarse en los trabajos presentados por Yang (2009), Singh y Singh (2012) y González et al. (2015). La presente investigación pretende evaluar la potencialidad como biopreservantes de la madera de Pinus caribaea var. hondurensis, de extractos etanolicos crudos del duramen de tres especies latífoliadas de elevada durabilidad natural en Venezuela, Handroanthus serratifolius, Centrolobium paraense y Tectona grandis. La mayor contribución de esta investigación será la apertura de nuevos caminos en la obtención de compuestos orgánicos con carácter preservante a partir de material natural, los cuales pueden ser empleado en la industria de la preservación de las maderas, disminuyendo así los riesgos o problemas toxicológicos generados en el manejo y uso de los preservantes tradicionales.

\section{MATERIALES Y MÉTODOS}

\section{Obtención de biopreservante}

Los extractos etanolicos del duramen de $H$. serratifolius, C. paraense y T. grandis se obtuvieron por procesos térmicos descritos por Velásquez et al. 
(2006). Chip del duramen de las especies estudiadas, fueron procesados en un molino Wiley mill y tamizados hasta obtener un tamaño de partículas adecuado para el proceso de extracción (60 Mesh). $30 \mathrm{~g}$. del material lignocelulosico molido por cada especie se colocó bajo reflujo en extractores Soxhlet con $300 \mathrm{ml}$ etanol (95\%) a $60{ }^{\circ} \mathrm{C}$ durante $8 \mathrm{~h}$. La mezcla disolvente/extractivos obtenida se concentró bajo vacío hasta sequedad en un rotaevaporador, para separar el solvente. El extracto seco se re-disolvió en etanol al 70 \% y se preparó una solución stock para dosificar las concentraciones de prueba $(0.2 \% 1 \%$ y $2 \%)$ la cual se almacenó en refrigeración hasta el momento de uso.

\section{Preservación de la madera}

Se emplearon bloques de albura de $P$. caribaea (1.9 cm de arista), libres de nudos, moho, manchas y exceso de resinas. Se emplearon un total de 72 bloques de madera por cada bio preservante 36 bloques por cada hongo, 12 bloques por concentración) y 16 bloques de control (8 tratados con etanol 70 \% y 8 tratados con agua). Todas las probetas fueron impregnadas mediante tratamiento por presión, siguiendo el procedimiento a célula vacía o Lowry sin la aplicación de vacío final. Los bloques de madera previamente secos y pesados, fueron sumergidos en la solución preservante $(0.2 \%, 1 \%$ y $2 \%$ ), colocados en autoclave y sometidos a un incremento de presión hasta $15 \mathrm{~kg} . \mathrm{cm}^{-2}$, mantenidos durante 20 minutos. Posterior al proceso de preservación, las muestras fueron extraídas de la solución preservante, retirado el exceso de líquido y mantenidas por $48 \mathrm{~h}$ a temperatura ambiente en bolsas de polietileno para permitir las reacciones entre la madera y los productos químicos. Las probetas fueron pesadas antes y después del tratamiento para determinar retención $\left(\mathrm{kg} \cdot \mathrm{m}^{-3}\right)$.

\section{Bioensayo}

Para evaluar la eficiencia del biopreservante, se siguió la metodología estandarizada por American
Society for Testing and Materials (2005a), en la norma ASTM D-1413. Se emplearon dos microorganismos de prueba: hongo de pudrición blanca Trametes versicolor (L: Fr) Pilát (FP-133255-R) y hongo de pudrición marrón Gloeophyllum trabeum (Fr.) Murr. (Mad-617-R). Se prepararon cámaras de deterioro de $500 \mathrm{~cm}^{3}$ de capacidad con suelo y placas de alimentación previamente esterilizadas, las cuales fueron asépticamente inoculadas y colonizadas por los microorganismos de prueba. Los bloques de madera, tratados y no tratados, fueron secados en estufa a $80 \pm 5{ }^{\circ} \mathrm{C}$ durante $24 \mathrm{~h}$, posteriormente esterilizados a $120{ }^{\circ} \mathrm{C}$ durante 15 minutos, enfriados en desecador y colocados sobre el micelio activo de los hongos en las cámaras de deterioro. Se expusieron a la acción degradante durante 16 semanas almacenadas en un cuarto acondicionado a $26 \pm$ $2{ }^{\circ} \mathrm{C}$ y $75 \pm 5 \%$ de humedad relativa. Después del periodo de incubación los bloques de madera fueron extraídos de las cámaras, cuidadosamente cepillado el micelio del hongo y secados en estufa a 80 $\pm 5{ }^{\circ} \mathrm{C}$ hasta obtener peso constante. La pérdida de peso de los bloques de madera se determinó gravimétricamente, al comparar la variación experimentada antes y después del bioensayo. El porcentaje de pérdida del peso seco de la madera fue usado como una medida de la resistencia al deterioro que presenta la madera al ser tratada con productos químicos y se determinó empleando la ecuación:

$$
P \mathrm{p}=\frac{P i-P f}{P i} \times 100
$$

$P \mathrm{p}=$ pérdida de peso $(\%), \mathrm{Pi}=$ peso seco inicial antes del ensayo $(\mathrm{g}), \mathrm{Pf}=$ peso seco al final del ensayo (g).

La pérdida de peso fue registrada y comparada con la clasificación presentada por la American Society for Testing and Materials (2005b), en la norma ASTM D-2017.

\section{Análisis estadístico}

Se empleó un modelo estadístico factorial $2 \times 3 \times$ 3 (microorganismo/tratamiento/concentración). La 
Tabla 1. Índices de durabilidad de la madera expresada como pérdida de peso y masa residual.

\begin{tabular}{ccl}
\hline Pérdida de peso $\mathbf{( \% )}$ & Masa residual promedio $\mathbf{( \% )}$ & \multicolumn{1}{c}{ Clases de resistencia } \\
\hline 0 a 10 & 90 a 100 & Altamente resistente \\
11 a 24 & 76 a 89 & Resistente \\
25 a 44 & 56 a 75 & Moderadamente resistente \\
45 superior & 55 o menos & $\begin{array}{l}\text { Levemente resistente } \\
\text { No resistente }\end{array}$ \\
\hline
\end{tabular}

Fuente: American Society for Testing and Materials (2005b).

data se analizó empleando el programa estadístico SPSS $^{\circledR}$ (19.0). Se evaluó la normalidad y homogeneidad de la varianza aplicando Shapiro-Wilk y el estadístico de Levene respectivamente. Se aplicó análisis de varianzas de un factor (Anova), y las pruebas de comparación de medias de Tukey para evaluar diferencias estadísticamente significativas entre los factores, a un nivel de significancia del $95 \%$.

\section{RESULTADOS}

En la figura 1 se muestran los niveles de retención promedio de los extractos etanólicos crudos del duramen de $H$. serratifolius, $C$. paraense y $T$. grandis empleados como biopreservantes sobre la madera de $P$. caribaea. El nivel de retención del biopreservante se incrementó en la medida que aumentó la concentración de la solución preservante desde $0.2 \%$ hasta $2 \%$, alcanzándose el mayor nivel de retención con el extracto de $H$. serratifolius, de $9.61 \mathrm{~kg} \cdot \mathrm{m}^{-3}$, y la menor retención con el extracto obtenido del duramen de C. paraense al $0.2 \%$ de concentración.

La pérdida de peso en la madera de albura de $P$. caribaea tratada y no tratada, bajo la acción degradante de hongos de pudrición marrón y blanca, se resumen en la tabla 2. Los resultados señalan que la madera de $P$. caribaea sin tratamiento muestra baja durabilidad natural en condiciones de laboratorio asociada a G. trabeum y T. versicolor, con pérdidas de $65.5 \%( \pm 1.11)$ y $55.3 \%( \pm 0.78)$ del

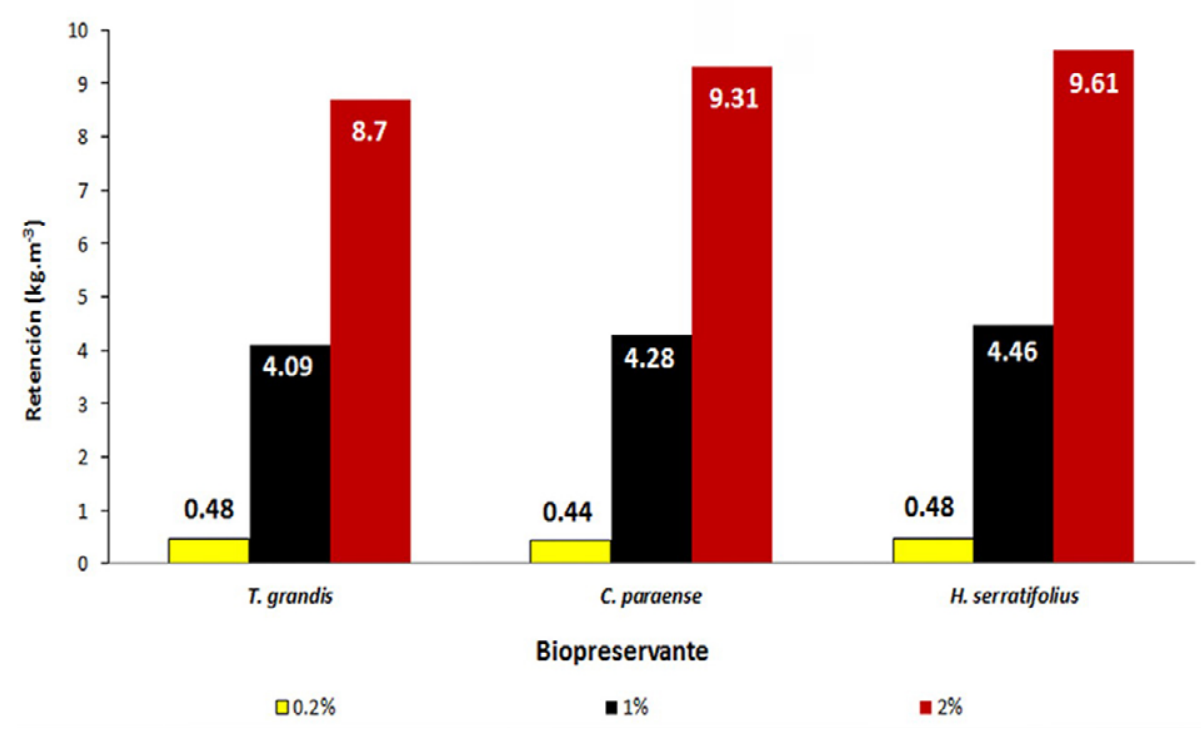

Figura 1. Niveles de retención de los biopreservantes en la madera de albura de $P$. caribaea. 
peso seco de la madera respectivamente. Los resultados evidencian la elevada actividad biológica de los microorganismos de prueba con las condiciones experimentales favorables.

Se indica valor promedio y desviación estándar en paréntesis. Las letras se leen horizontalmente. Letras diferentes dentro de cada microorganismo y tratamiento indican diferencias estadísticas ( $p$ $<0.05$ ) de acuerdo a la prueba de Tukey. $n=12$ muestras por concentración. 16 semanas de evaluación.

Los bloques de madera sin tratamiento expuestos a los microorganismos de prueba durante 16 semanas mostraron extensivos signos de deterioro (decoloración de la madera y colonización completa de la superficie de la probeta); en cambio, en los bloques tratados estos signos fueron menos evidentes. El análisis estadístico (Anova) y comparación de medias de Tukey demuestran diferencias altamente significativas en la pérdida de peso, entre las probetas de $P$. caribaea tratadas y no tratadas $\left(F_{1,232}=271.4, p<0.001\right)$ así como entre la variable concentración del biopreservante $\left(F_{3,232}=295.7\right.$, $p<0.001$ ) en cada tratamiento (tabla 2 y figura 2 ). Estos resultados evidencian que el tratamiento de la madera con los biopreservantes, incrementan significativamente la resistencia al deterioro asociada a T. versicolor y G. trabeum.
Los tratamientos evaluados (biopreservantes) muestran mayor eficacia en la inhibición de la actividad degradante sobre hongos de pudrición blanca que sobre hongos de pudrición marrón. G. trabeum, resulto ser más agresivo que T. versicolor, ocasionando una pérdida de peso significativamente mayor $\left(\mathrm{F}_{1,232}=8.25, p=0.003\right)$, sobre la madera tratada y las no tratadas. Estos resultados evidencian la preferencia de los hongos de pudrición marrón sobre las especies coníferas. El comportamiento descrito se observó en todas las concentraciones evaluadas y dentro de cada tratamiento (tabla 2 y figura 2).

En la tabla 3 se muestran los resultados del Anova y comparación de medias de Tukey al $95 \%$ de confianza, de la pérdida del peso de $P$. caribaea bajo los tres tratamientos al $2 \%$ de concentración del biopreservante. La pérdida de peso experimentada por la madera de $P$. caribae varió entre $17 \%$ y $26 \%$ frente a G. trabeum y entre $13 \%$ y $25 \%$ frente a $T$. versicolor, dependiendo del tipo de biopreservante y el nivel de retención $\left(8.70 \mathrm{~kg} \cdot \mathrm{m}^{-3}\right.$ y $\left.9.31 \mathrm{~kg} \cdot \mathrm{m}^{-3}\right)$. El análisis estadístico reveló diferencias altamente significativas entre los tratamientos evaluados $(p<0.001)$, evidenciándose en la formación de cuatro grupos estadísticamente diferentes, frente a cada microorganismo de prueba (tabla 3).

Tabla 2. Pérdida de peso de la madera de $P$. caribaea frente a G. trabeum y $T$. versicolor, tratada con extractos etanólicos del duramen de $H$. serratifolius, C. paraense y $T$. grandis a tres niveles de concentración $(0.2 \%, 1 \%$ y $2 \%$ p/v)

\begin{tabular}{|c|c|c|c|c|c|c|}
\hline \multirow{3}{*}{ Extract etanolic } & \multicolumn{3}{|c|}{ Gloeophyllum trabeun } & \multicolumn{3}{|c|}{ Trametes versicolor } \\
\hline & \multicolumn{3}{|c|}{ Concentración (\%) } & \multicolumn{3}{|c|}{ Concentración (\%) } \\
\hline & 0.2 & 1 & 2 & 0.2 & 1 & 2 \\
\hline H. serratifolius & $\begin{array}{l}34.30 \mathrm{~A} \\
( \pm 3.09)\end{array}$ & $\begin{array}{l}22.67 \mathrm{~B} \\
( \pm 2.32)\end{array}$ & $\begin{array}{l}17.86 \mathrm{C} \\
( \pm 1.81)\end{array}$ & $\begin{array}{l}25.30 \mathrm{~A} \\
( \pm 1.89)\end{array}$ & $\begin{array}{l}19.42 \mathrm{~B} \\
( \pm 1.06)\end{array}$ & $\begin{array}{l}13.14 \mathrm{C} \\
( \pm 1.31)\end{array}$ \\
\hline $\begin{array}{l}\text { Centrolobium } \\
\text { paraense }\end{array}$ & $\begin{array}{l}41.28 \mathrm{~A} \\
( \pm 1.37)\end{array}$ & $\begin{array}{l}32.05 \mathrm{~B} \\
( \pm 1.10)\end{array}$ & $\begin{array}{l}23.03 \mathrm{C} \\
( \pm 1.33)\end{array}$ & $\begin{array}{l}37.92 \mathrm{~A} \\
( \pm 1.43)\end{array}$ & $\begin{array}{l}28.43 \mathrm{~B} \\
( \pm 1.18)\end{array}$ & $\begin{array}{l}21.40 \mathrm{C} \\
( \pm 1.21)\end{array}$ \\
\hline Tectona grandis & $\begin{array}{l}43.16 \mathrm{~A} \\
( \pm 1.33)\end{array}$ & $\begin{array}{l}35.19 \mathrm{~B} \\
( \pm 2.19)\end{array}$ & $\begin{array}{l}26.98 \mathrm{C} \\
( \pm 0.83)\end{array}$ & $\begin{array}{l}39.75 \mathrm{~A} \\
( \pm 0.55)\end{array}$ & $\begin{array}{l}30.11 \mathrm{~B} \\
( \pm 2.00)\end{array}$ & $\begin{array}{l}25.02 \mathrm{C} \\
( \pm 0.01)\end{array}$ \\
\hline Control & & $\begin{array}{l}65.55 \mathrm{D} \\
( \pm 1.11) \\
\end{array}$ & & & $\begin{array}{l}55.37 \mathrm{D} \\
( \pm 0.78) \\
\end{array}$ & \\
\hline
\end{tabular}

Se indica valor promedio y desviación estándar en paréntesis. Las letras se leen horizontalmente. Letras diferentes dentro de cada microorganismo y tratamiento indican diferencias estadísticas $(\mathrm{p}=0.05)$ de acuerdo a la prueba de Tukey. $\mathrm{n}=12$ muestras por concentración. 16 semanas de evaluación 


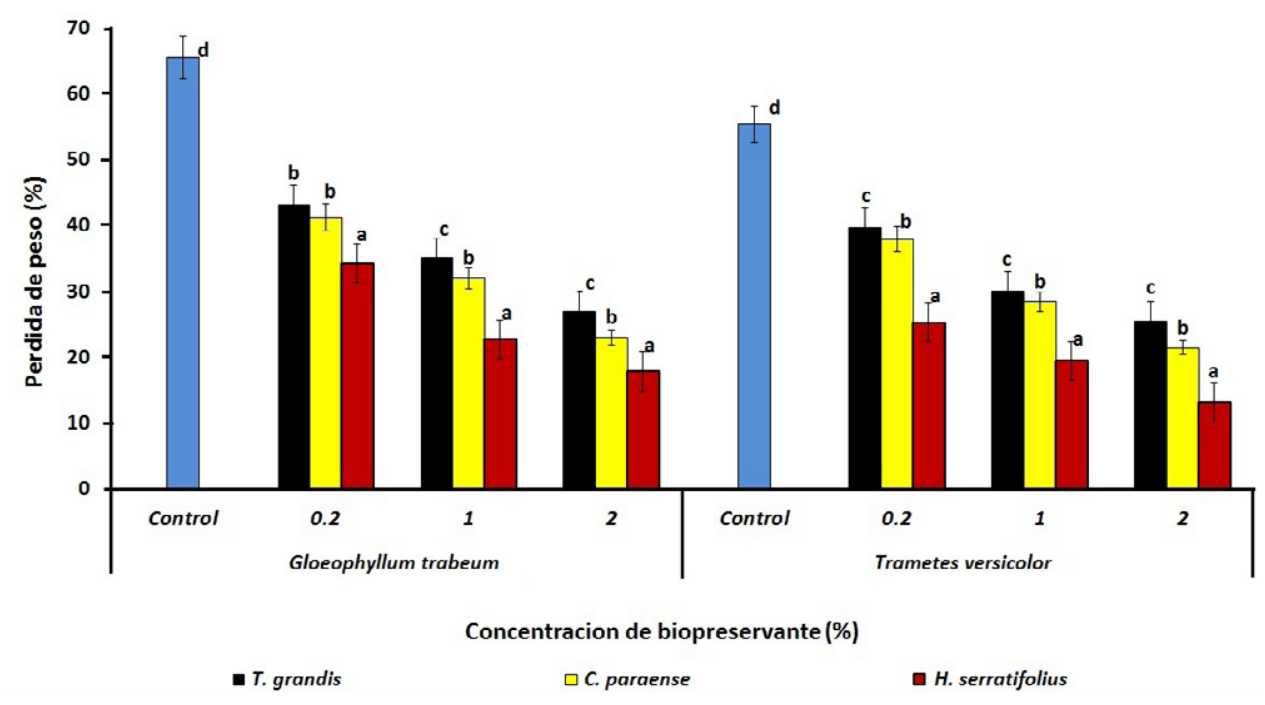

Figura 2. Pérdida de peso de bloques de P.caribaea tratados con el extracto etanólico de diferentes especies y expuestos a T. versicolor y G. trabeum. Barras con diferentes letras difieren significativamente $(p<0.05)$. Para cada concentración extracto/hongo $n=12$.

Tabla 3. Análisis de varianza y comparación de medias de la pérdida de peso entre tratamientos $2 \%$ de concentración sobre la madera de P. caribae, frente a G. trabeum y T. versicolor.

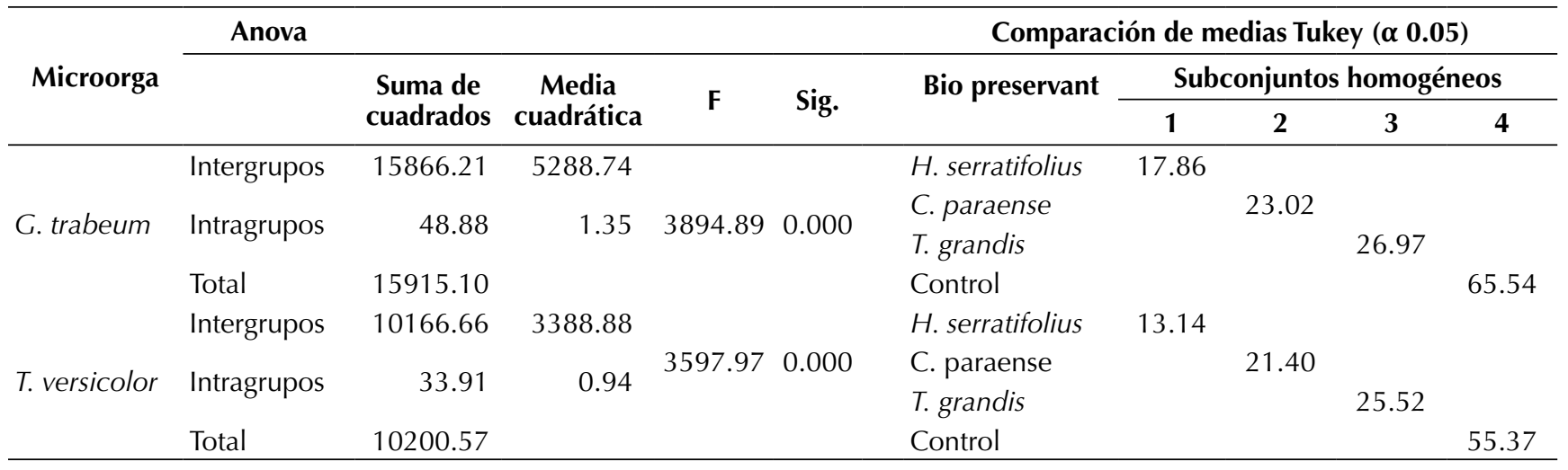

Si bien todos los tratamientos mostraron diferentes niveles de protección sobre la madera de $P$. caribaea, el tratamiento con extracto de $H$. serratifolius con el mayor nivel de retención (9.61 kg. m³) mostró el mejor comportamiento frente a G. trabeum y $T$. versicolor, con una pérdida de peso promedio por debajo del $18 \%$. Las probetas tratadas con esta solución experimentaron una reducción de la pérdida de peso cuatro veces menor en comparación con las muestras no tratadas. El tratamiento con extracto C. paraense mostró una efectividad moderada en comparación con los otros dos tratamientos, evidenciando una pérdida del peso tres veces menor en comparación con las muestras control. La madera tratada con el extracto de T. grandis mostró el menor nivel de protección con pérdida de peso promedio del $26 \%$, superior a la observada con los extractos de $H$. serratifolius y $C$. paraense. 


\section{DISCUSIÓN}

Los resultados obtenidos en la investigación señalan que la madera de $P$. caribaea sin tratamiento evidenció los mayores niveles de deterioro y pérdida de peso frente G. trabeum y T. versicolor. HaIlazgos similares sobre la baja durabilidad natural de la madera de $P$. caribaea han sido previamente reportados. Un ejemplo de ello son los trabajos de Mora y Encinas (2001), Ramírez et al. (2005) y Gómez et al. (2011), quienes señalaron perdidas de peso cercanos al $60 \%$ frente a G. trabeum y $T$. versicolor.

La menor pérdida de peso causada por T. versicolor en comparación con G. trabeum sobre la madera de $P$. caribaea, puede estar relacionada con la habilidad propia del microorganismo, ya que los hongos de pudrición blanca son más eficientes en la degradación de la madera de especies latífoliadas en comparación con la madera de coníferas (Pant y Tripathi, 2011; Broco et al., 2017), tal es el caso de la especie evaluada en esta investigación.

La madera de $P$. caribaea tratada con los diferentes extractos mostró un incremento significativo de su resistencia frente a hongos de deterioro; estos resultados son similares a los reportados en la literatura especializada, en los cuales se evidencia la actividad biológica de los extractos naturales sobre los microorganismos que deterioran la madera. Onuorah (2000) demostró la eficacia del extracto metanolico del duramen de especies latífoliadas como preservante de la madera sobre hongos de pudrición blanca y marrón. Tumen et al. (2013) determinaron la eficiencia del tratamiento de la madera (spruce/pine/fir) con el extracto etanolico del duramen de tres especies de Juniperus en el control de G. trabeum y T.versicolor. Eller et al. (2018) reportaron la actividad biológica sobre G. trabeum y T.versicolor del aceite de Juniperus virginiana impregnado en la madera de spruce, pine y fir. Por otro lado, Kwaśniewska-Sip et al. (2018) incrementaron la resistencia de la madera de Pinus sylvestris al tratarla con extracto de cafeína frente a T.versicolor.
Los biopreservantes evaluados mostraron excelentes resultados en pruebas de toxicidad in vitro (100\% inhibición de desarrollo), a una concentración de $0.2 \%$, utilizando agar-extracto de malta frente a G. trabeum y $T$. versicolor (Velásquez et al., 2006). Sin embargo, cuando estos extractos a igual concentración se introdujeron en la microestructura de la madera de $P$. caribaea su actividad fue menor, posiblemente debido a la lixiviación o volatilización del producto, así como una consecuencia de la resultante interacción extracto/ madera (Eaton y Hale, 1993). De acuerdo con Syofuna et al. (2012), el efecto protector de los extractos del duramen empleados como preservantes sobre la madera de $P$. caribaea puede atribuirse a factores relacionados con la complejidad y variedad de los compuestos químicos que integran estos extractivos y a la elevada permeabilidad de la madera de $P$. caribaea que permite alcanzar elevados niveles de retención y protección.

Un incremento en la concentración de la solución tratante se reflejó en una mejora de la resistencia al deterioro de $P$. caribaea, estos resultados son similares a los obtenidos sobre otras coníferas. Tascioglu et al. (2013) impregnaron la madera de Pinus sylvestris con extractos acuoso de corteza y duramen de Acacia mollissima y Schinopsis lorentzii respectivamente, a diferentes niveles de concentración (3\%, $6 \%$, 9 \% y $12 \%$ ). Sus resultados evidenciaron la misma tendencia, es decir, un incremento significativo de la eficacia del biopreservante en la medida que se incrementó la concentración del mismo frente a T. versicolor y G. trabeum, obteniéndose los mejores resultados antifúngicos con las muestras tratadas al $9 \%$ y $12 \%$ de concentración, niveles de 4 y 6 veces superiores al máximo evaluado en esta investigación, lo que demuestra la eficacia de los extractos aquí evaluados.

Al comparar los resultados con otras investigaciones en la que se busca incrementar la durabilidad de la madera de $P$. caribaea, se encontró que Mora y Encinas (2001) evaluaron la durabilidad inducida de la madera de esta especie tratada con 
sales CCA al $5 \%$ de concentración. Sus resultados señalaron pérdidas de peso inferior al $1 \%$, obviamente la eficacia de este producto es superior a los obtenidos en esta investigación. Sin embargo, es importante destacar la eficacia de estos extractos naturales en el control del deterioro biológico de la madera de $P$. caribaea, los cuales fueron empleados a menor concentración, crudos y sin purificación o fraccionamiento de sus ingredientes activos.

La elevada actividad biológica del biopreservante de $H$. serratifolius obtenida en esta investigación es similar a la señalada por Rodrigues et al. (2012), quienes determinaron la eficacia del extracto metanolico del duramen $H$. serratifolius sobre albura de Pinus sylvestris frente a G. trabeum. El uso del extracto etanolico de $T$. grandis como preservante para madera ha sido reportado por Adegeye et al. (2009) y Broco et al. (2017); sobre la madera de Gmelina arborea, Triplochiton scleroxylo y Pinus sp. respectivamente, al igual que en esta investigación, sus resultados evidenciaron la eficacia del extracto en el control de hongos de pudrición blanca. Es importante resaltar que la eficacia obtenida en esas investigaciones se logró a una concentración superior (4\%) a la máxima evaluada en esta investigación.

Estudios previos han señalado que la actividad antifúngica y protectora de los extractos del duramen de $H$. serratifolius y $T$. grandis, frente a hongos que deterioran la madera, está asociada a la presencia de compuestos de tipo quinonas en su composición, como naftoquinonas, antraquinonas y tectoquinonas (Velásquez et al., 2004; Kokutse et al., 2006; Thulasidas y Bhat, 2007; Nagadesi y Arya, 2013; Broco et al., 2016). Es necesario continuar con las investigaciones en el aislamiento, elucidación y citotoxicidad de los ingredientes activos presentes en el extracto de C. paraense para ser empleados en la industria de la preservación de la madera en la formulación de nuevos productos químicos ambientalmente amigables.

Considerando el sistema de clasificación de la ASTM (2005) basado en la pérdida de peso, la madera de $P$. caribaea sin tratamiento, es clasificada como una madera de baja durabilidad natural o no resistente al deterioro (tabla 1). Sin embargo, con la aplicación de los extractos crudos como biopreservantes, la madera de $P$. caribaea supera la clasificación de durabilidad, es decir, pasa a ser una madera moderadamente resistente con el tratamiento del extracto de $T$. grandis y una madera resistente al deterioro con el tratamiento de los extractos de $H$. serratifolius y $C$. paraense.

\section{CONCLUSIÓN}

Los extractos de origen natural pueden ser una alternativa importante para la industria de la protección de la madera, proporcionando biomoléculas capaces de ofrecer resistencia a la descomposición contra hongos, así como baja toxicidad sobre los seres humanos y el ambiente. Los resultados claramente demostraron la eficacia del tratamiento con extractos etanolicos del duramen de especies de elevada durabilidad natural, evidenciado en un incremento significativo de la resistencia al deterioro de la madera de $P$. caribaea frente a G. trabeum y $T$. versicolor. Los extractos evaluados mostraron bioactividades importantes sin ningún fraccionamiento o purificación previa. Al comparar los extractos se observó que el obtenido de $H$. serratifolius resultó ser el más efectivo en reducir el deterioro de la madera de $P$. caribaea, seguido del extracto de C. paraense y, con efectos más sutiles pero eficiente, el extracto de T. grandis. Continúan las investigaciones sobre el aislamiento, elucidación y citotoxicidad de las biomoléculas responsables de la actividad biológica en estos extractos.

\section{AGRADECIMIENTOS}

Los autores expresan su agradecimiento al Fondo Nacional de Ciencia y Tecnología (Fonacit), Caracas, Venezuela (Pem 2001001639) y Provita Caracas, Venezuela (2008-17) por su apoyo financiero. 


\section{CONFLICTO DE INTERESES}

Los autores declaran no tener conflicto de intereses.

\section{CONTRIBUCIÓN POR AUTOR}

J.V., L.R. y O. E. planearon y diseñaron la investigación, coordinaron y llevaron a cabo la investigación en el terreno, analizaron los datos y redactaron el manuscrito, supervisaron la investigación. Todos los autores siguieron las discusiones y comentaron los borradores anteriores.

\section{REFERENCIAS}

Adegeye, A., Ogunsanwo, O. y Olajuyigbe, S. (2009). Antifungal Activities of Heart Wood Extract (HWE) of Teak Tectona grandis Against Two White Rots in Woods of Gmelina arborea and Triplochiton scleroxylon. Academic Journal of Plant Sciences, 2(4), 279-285.

https://doi.org/10.4314/ijbcs.v4i3.60453

American Society for Testing and Materials (2005a). Standard Test Method for Wood Preservatives by Laboratory Soil-block Cultures. ASTM D-1413. Annual Book of ASTM Standard, Philadelphia.

American Society for Testing and Materials (2005b). Standard Method of Accelerated Laboratory Test of Natural Decay Resistance of Woods. ASTM D-2017. Annual Book of ASTM Standard, Philadelphia.

Antwi, C. y Damoah, A. (2010). Investigation of synergistic effects of extracts from Erythrophleum suaveolens, Azadirachta indica, and Chromolaena odorata on the durability of Antiaris toxicaria. International Biodeterioration \& Biodegradation, 64(2), 97-103.

https://doi.org/10.1016/j.ibiod.2009.08.005

Brocco, V., Paes, J., Gonçalves L., Brazolin S. y Chaves, M. (2017). Potential of teak heartwood extracts as a natural wood preservative. Journal of Cleaner Production, 142(4), 2093-2099.

https://doi.org/10.1016/j.jclepro.2016.11.074

Dawson, B. y Morrell, J. (1990). Effects of chemical pretreatment of douglas-fir hearwood on efficacy of potential bioprotection agents. The International Research Group on Wood Preservation. Document $\mathrm{N}^{\circ}$ IRG/WP/1440.

Doppelreiter, H. y Korioth, M. (1978). Inhibition of development of the subterranean termites Hetertermes indicola and Reticulitermes Flavipes caused by diflubenzuron (Dimilin ${ }^{R}$ ). Holzforschung, 32, 103-109.

https://doi.org/10.1111/j.1439-0418.1981.tb04461.x

Eaton, R. y Hale, M. (1993). Wood: Decay, Pests and Protection. Londres: Chapman \& Hall.

Eller, F., Hay, W., Kirker, G., Mankowski, M. y Sellling, G. (2018). Hexadecyl ammonium chloride amylose inclusion complex to emulsify cedarwood oil and treat wood against termites and wood-decay fungi. International Biodeterioration \& Biodegradation, 129(4), 95-101.

https://doi.org/10.1016/j.ibiod.2018.01.010

Fengel, D. y Wegener, G. (1984). Wood: Chemistry, UItrastucture and reactions. Brelín y Nueva York: W. de Gruyter.

Goktas, O., Mammadov, R., Duru, M., Ozen, E., Colak, M. y Yilmaz, F. (2007). Introduction and evaluation of the wood preservative potentials of the poisonous Sternbergia candidum extracts. African Journal of Biotechnology, 6(8), 982-986.

https://doi.org/10.1016/j.jbiotec.2008.07.1558

Gómez, L., Díaz, M., Velásquez, J., Toro, M., Márquez, A. y Castro, F. (2011). Resistencia natural al deterioro de especies latifoliadas de la Guayana Venezolana. Revista Copérnico, 7(14), 5-11.

https://doi.org/10.21829/myb.2006.1211250

González, R., Rosales, M., Rocha, N., Gallegos, J., Moreno, M. y Karchesy, J. (2015). Wood preservation using natural products. Madera y Bosques, 21(Núm. esp.), 63-76.

https://doi.org/10.21829/myb.2015.210427

Hillis, W. (1987). Heartwood and tree exudates. Nueva York: Syracuse.

Kamdem, D. (1994). Fungal decay resistance of aspen blocks treated with heartwood extracts. Forest Products Journal, 44(1), 30-32.

Kokutse, A., Stokes, A., Bailleres, H., Kokou, K. y Baudasse, Ch. (2006) Decay resistance of Togolese teak 
(Tectona grandis L.f) heartwood and relationship with color. Trees, 20(2), 219-223.

https://doi.org/10.1007/s00468-005-0028-0

Kwaśniewska-Sipa, P., Coftaa G. y Nowak, P. (2018). Resistance of fungal growth on Scots pine treated with caffeine. International Biodeterioration \& Biodegradation, 132(7), 178-184. DOI: https://doi.org/10.1016/j.ibiod.2018.03.007

Mora, N. y Encinas, O. (2001). Evaluación de la durabilidad natural e inducida de Pterocarpus acapulcensis, Tabebuia serratifolia y Pinus caribaea, en condiciones de laboratorio. Revista Forestal Venezolana, 45(1), 23-31.

Nagadesi, P. y Arya, A. (2013). Delignification pattern of wood decay by white rot fungi in teak (Tectona grandis L. f.). Journal of the Indian Academy of Wood Science, 10(1), 1-8.

https://doi.org/10.1007/s13196-013-0085-8

Onuorah, E. (2000). The wood preservative potentials of heartwood extracts of Milicia excelsa and Erythrophleum suaveolens. Bioresource Technology, 75(2), 171-173.

https://doi.org/10.1016/S0960-8524(99)00165-0

Pant, H. y Tripathi, S. (2011). Fumigation of wood with aluminium phosphide for protection against fungi. Journal of Tropical Forest Science, 23(4), 363-370.

Peredo, M. (1993). Preservantes antimancha alternativo al pentaclorofenato de sodio. Chile Forestal. Documento técnico $\mathrm{N}^{\circ} 68$.

Ramírez, E., Castro, F. y Velásquez, J. (2005). Durabilidad natural de la madera de Baraman (Catostemma commune Sandwith). Revista Copérnico, 2(3), 269-272.

Rodrigues, A., Stien, D., Eparvier, V., Espindola, L., Beauchêne, J., Amusant, N., Leménager, N., Baudassé, Ch. y Raguin, L. (2012). The wood preservative potential of long-lasting Amazonian wood extracts. International Biodeterioration \& Biodegradation, 75(10), 146-149.

https://doi.org/10.1016/j.ibiod.2012.03.014

Sa'R., Argolo, A., Napoleẫo, T., Gomes, F., Santos, N., Melo, C., Albuquerque, A., Xavier, H., Coelho, L., Bieber, L. y Paiva, P. (2009). Antioxidant, Fusarium growth inhibition and Nasutitermes corniger repellent activities of secondary metabolites from Myracrodruon urundeuva heartwood. International Biodeterioration \& Biodegradation, 63(4), 470-477.

https://doi.org/10.1016/j.ibiod.2009.01.002

Salem, M., Zidan, E., Mansour, M. y Abo, W. (2016). Evaluation of usage three natural extracts applied to three commercial wood species against five common molds. International Biodeterioration \& Biodegradation, 110, 206-226.

https://doi.org/10.1016/j.ibiod.2016.03.028

Schultz, T. y Nicholas, D. (2000). Naturally durable heartwood: evidence for a proposed dual defensive function of the extractives. Phytochemistry, 54(1), 47-52.

https://doi.org/10.1016/S0031-9422(99)00622-6

Schultz, T. y Nicholas, D. (2002). Development of environmentally-benign wood preservatives based on the combination of organic biocides with antioxidants and metal chelators. Phytochemistry, 61(5), 555-560.

https://doi.org/10.1016/S0031-9422(02)00267-4

Sen, S., Tascioglu, C. y Tirak, K. (2009). Fixation, leachibility and dacay resistance of Wood treated with some comercial extracts and Wood preservatives salts. International Biodeterioration \& Biodegradation, 63(2), 135-141.

https://doi.org/10.1016/j.ibiod.2008.07.007

Singh, T. y Singh, A. (2012). A review on natural products as wood protectant. Wood Science and Technology, 46(5), 851-870.

https://doi.org/10.1007/s00226-011-0448-5

Sjöström, E. (1981). Wood Chemistry Fundamental and Applications. Orlando, Florida, EE. UU.

Suttie, E. y Orsler, R. (1996). The influence of the natural extractives of Opepe (Nauclea diderrichii) and African padauk (Pterocarpus soyauxii) timbers on their durability. The International Research Group on Wood Preservation. Document No IRG/ WP/ 96-30098.

Syofuna, A., Banana, A. y Nakabonge, G. (2012). Efficiency of natural wood extractives as wood preservatives against termite attack. Maderas Ciencia y Tecnología, 14(2), 155-163.

https://doi.org/10.4067/s0718-221×2012000200003 
Tascioglu C., Yalcin, M., Sen, S. y Akcay, C. (2013). Antifungal properties of some plant extracts used as wood preservatives. International Biodeterioration \& Biodegradation, 85(1), 23-28.

https://doi.org/10.1016/j.ibiod.2013.06.004

Thulasidas, P. y Bhat, K. (2007). Chemical extractive compounds determining the brown-rot decay resistance of teakwood. Holz als Roh-und Werkstoff, 65, 121-124.

https://doi.org/10.1007/s00107-006-0127-7

Tsoumis, G. (1991). Science and technology of wood. structure, properties, utilization. Nueva York: Van Nostrand Reinhold.

Tumen, I., Eller F., Clausen C. y Teel, J. (2013). Antifungal Activity of Heartwood Extracts from Three Juniperus species. BioResources, 8(1), 12-20.

https://doi.org/10.15376/biores.8.1.12-20
Velásquez, J., Rojas, L. y Usubillaga, A. (2004). Antifungal activity of naphtoquinone from Tabebuia serratifolia (Vahl. Nicholson). Ciencia, 12(1), 64-69.

Velásquez, J., Toro, M., Rojas, L. y Encinas, O. (2006). Actividad antifúngica in vitro de los extractivos naturales de especies latífoliadas de la Guayana Venezolana. Madera y Bosques, 12(1), 51-61.

https://doi.org/10.21829/myb.2006.1211250

Yang, D. (2009). Potential utilization of plant and fungal extracts for wood protection. Forest Products Journal, 59(4), 97-103. 